\title{
Iterative Estimation for Flight Dynamic Helicopter Simulator
}

Ivana Yoshie Sumida ${ }^{1,2}$, Haroldo F. de Campos Velho ${ }^{3}$, Eduardo F. P. da Luz ${ }^{3}$, Ronaldo V. Cruz ${ }^{4}$ and Luiz Carlos S. Góes ${ }^{4}$

1. Applied Computing Graduate Program, National Institute for Space Research (INPE), Brazil

2. Flight Test Research Institute (IPEV), Brazil

3. Associated Laboratory for Computing and Applied Mathematics, National Institute for Space Research (INPE), Brazil

4. Department of Mechanical Engineering, Technological Institute of Aeronautics (ITA), Brazil

Received: August 19, 2015 / Accepted: September 17, 2015 / Published: December 25, 2015.

\begin{abstract}
Flight simulators can provide a suitable alternative to real flight, mainly to increase safety through the training of crew, and evaluation data from simulator can be used to validation and certification of aircraft systems. However, it must convey some degree of realism to the user to be effective. For that reason, it is necessary to calibrate the simulator software. Calibration for flight simulation is parameter identification process. The process is formulated as an optimization problem, and it is solved by using a new approach named Multiple Particle Collision Algorithm (MPCA). Results show a good performance for the employed approach.
\end{abstract}

Key words: Dynamic Flight, Parameter Identification, Multiple Particle Collision Algorithm

\section{Introduction}

The dynamics of flight are critical for aircraft design, looking at the adaptation of automatic control systems and flight simulators. Identification techniques applied to dynamics flight has had an improvement in the increasing availability of faster computers.

The identification of parameters methodology has applications in several areas such as: astronomy, aerospace, economics, biology, electrical, geological, etc $[1,2,3]$. The strategy is to adjust the unknown parameters in order to have the best fit of a mathematical model for a phenomenon with the observations. Tools and techniques of identification have evolved to match the complexity and the increasing need for correction and precision in the results. This methodology is more accurate than the

Corresponding author: Ivana Yoshie Sumida, Applied Computing Graduate Program, National Institute for Space Research (INPE), Brazil. E-mail: ivanayoshie@yahoo.com.br. corresponding values predicted by other methods such as analytical and numerical differentiation $[4,5]$. Thus, identification of parameters has become a significant tool for applications such as model validation, handling qualities evaluation, control law design, and flight-vehicle design and certification [6].

Relating to helicopter system identification techniques, few articles have used stocasthic methods for global optimization of a cost function based on the prediction error. We can cite Cruz et al. $[7,8]$ in the longitudinal mode system identification of the Twin Squirrel helicopter and Cerro [9] in the identification of a small unmanned helicopter model.

Parameter identification associated with the aerodynamic performance of the aircraft can be formulated as an optimization problem. In this paper, a new meta-heuristics, named multiple particle collision algorithm (MPCA) [10], was applied to the calibration of a dynamic flight simulator. The MPCA optimization algorithm was inspired from some typical phenomena inside of nuclear reactors during the neutron travel: 
absorption and scattering of multiple particles. The results obtained with MPCA are compared to the ones obtained by Cruz [12], where Genetic Algorithm (GA) was used to find the parameters.

\section{Parameter Identification Methodology}

The methodology for the identification of parameters used in this work is the well-known Quad-M, proposed by Jategaonkar [13]. This methodology takes into account the main elements of rotorcraft system identification, including the rotorcraft excitation maneuvers, the aerodynamic data measurements, the mathematical model of the helicopter equations of motion, and the parameter estimation methods used to minimize the predicted output-error between the model and the real data, as shown in Figure 1. Here it is important to notice that the method used was MPCA. Each one of these elements will be discussed below.

\subsection{Maneuver}

The dynamic response and results from the application of inputs such as pulse, step, doublet, multistep, sinusoidal, 3-2-1-1, among others. Thus, a wide variety of maneuvers can be specified. We considered the following basic principle in identification systems: the data records of the flight test must contain the information of the dynamic characteristics to be obtained in the model [12]. In this work, only the sinusoidal frequency input will be used.

\subsection{Measurements}

The tested helicopter was equipped with the Aydin Vector Data Acquisition System (AVDAS) PCU-816-I, ATD-800 digital recorder, this system measures a total of thirty-five different parameters. Some of the measured data channels include fuel quantity in each tank, nose boom static and dynamic pressures, external stagnation temperature, aerodynamic angle of attack $(\alpha)$ and sideslip $(\beta)$, roll, pitch, and yaw rates ( $p, q$ and $r$, resp.), load factors, longitudinal $(\theta)$ and lateral $(\varphi)$ body attitudes, heading, collective, longitudinal and lateral cyclic, and pedal command deflections $\left(\delta_{c}, \delta_{b}, \delta_{a}\right.$ and $\delta_{p}$, resp.) .

The Earth axis speeds $(u, v, w)$ are obtained with the aid of a Z12 Differential Global Positioning System DGPS, from Astech, whose antenna is fixed in the top of the vertical fin. The DGPS and AVDAS data synchronization is made by inserting a simultaneous

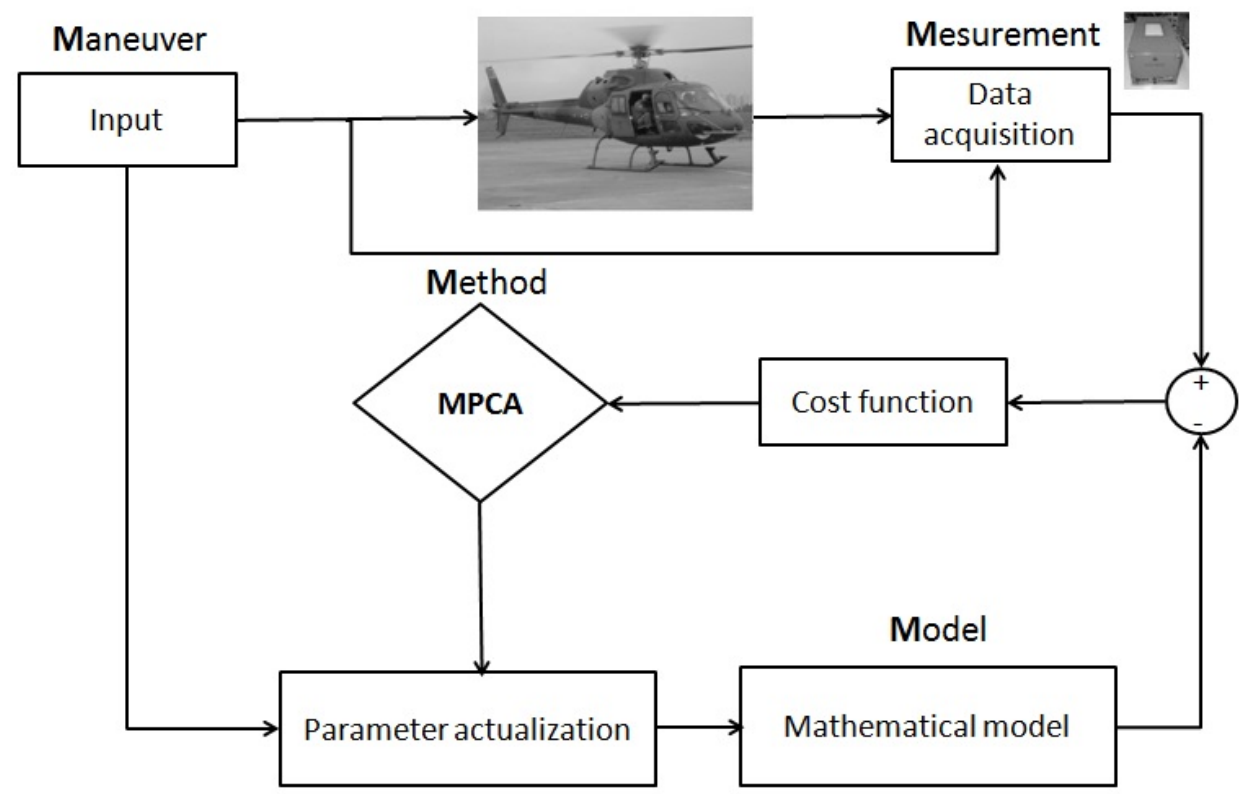

Fig. 1 Adaptation Quad-M Methodology. 
event in both systems. The DGPS data is represented with the same AVDAS data sampling rate by means of linear interpolation procedure.

The wind direction and intensity are obtained comparing the body axis speeds with the aerodynamic speed from the flight-test air data system, mounted on a nose boom, at trim conditions. Consequently, the body axis speeds $(u, v, w)$ are easily calculated adding wind vector to the Earth axis speeds [14].

\subsection{Linearized Model Flight Dynamic}

The helicopter equations of motion, deduced from the Newton second law for translational and rotational movements are given by [15] and [16] as:

$$
X=m(\dot{u}-r v+q w)+m g \sin \theta
$$

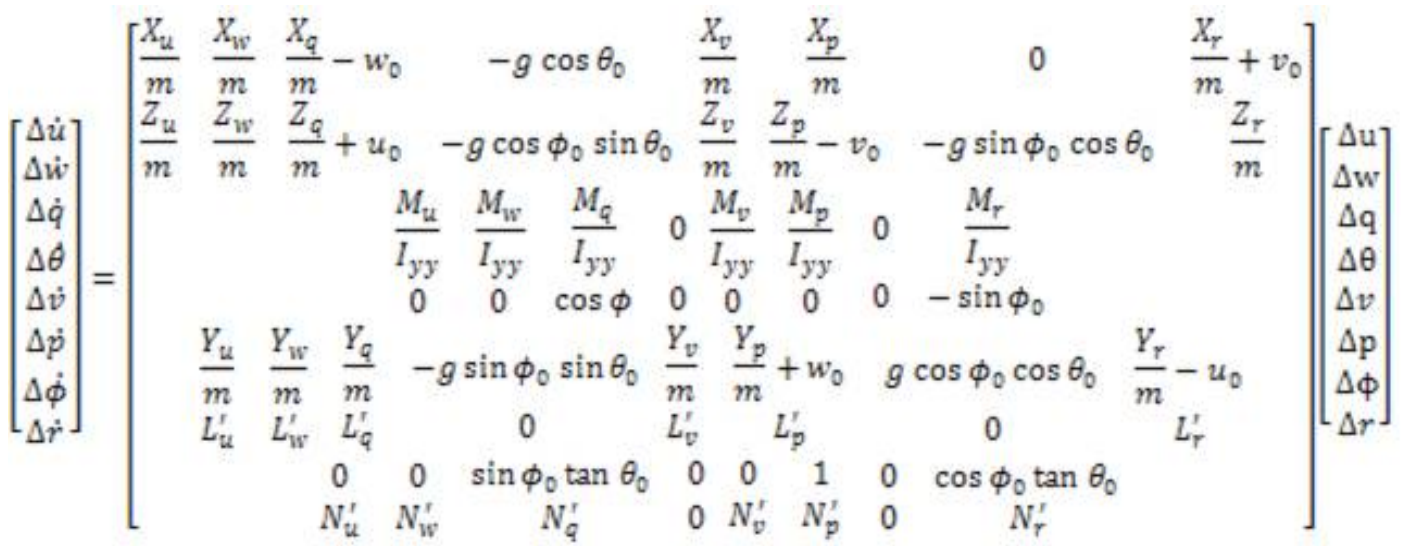

$$
\begin{aligned}
& +\left[\begin{array}{cccc}
\frac{X_{\delta_{b}}}{m} & \frac{X_{\delta_{c}}}{m} & \frac{X_{\delta_{a}}}{m} & \frac{X_{\delta_{p}}}{m} \\
\frac{Z_{\delta_{b}}}{m} & \frac{Z_{\delta_{\varepsilon}}}{m} & \frac{Z_{\delta_{a}}}{m} & \frac{Z_{\delta_{p}}}{m} \\
\frac{M_{\delta_{b}}}{I_{y y}} & \frac{M_{\delta_{\varepsilon}}}{I_{y y}} & \frac{M_{\delta_{a}}}{I_{y y}} & \frac{M_{\delta_{p}}}{I_{y y}} \\
0 & 0 & 0 & 0 \\
\frac{Y_{\delta_{b}}}{m} & \frac{Y_{\delta_{\varepsilon}}}{m} & \frac{Y_{\delta_{a}}}{m} & \frac{Y_{\delta_{p}}}{m} \\
L_{\delta_{b}}^{\prime} & L_{\delta_{\varepsilon}}^{\prime} & L_{\delta_{a}}^{\prime} & L_{\delta_{p}}^{\prime} \\
0 & 0 & 0 & 0 \\
N_{\delta_{b}}^{\prime} & N_{\delta_{\varepsilon}}^{\prime} & N_{\delta_{a}}^{\prime} & N_{\delta_{p}}^{\prime}
\end{array}\right]\left[\begin{array}{l}
\delta_{b} \\
\delta_{c} \\
\delta_{a} \\
\delta_{p}
\end{array}\right]
\end{aligned}
$$

$$
\begin{aligned}
& Y=m(\dot{v}-p w+r u)-m g \cos \theta \sin \phi \\
& Z=m(\dot{w}-q u+p v)-m g \cos \theta \cos \phi \\
& L=I_{x x} \dot{p}-I_{z x}(\dot{r}+p q)-\left(I_{y y}-I_{z z}\right) q r \\
& M=I_{y y} \dot{q}-I_{z x}\left(r^{2}-p^{2}\right)-\left(I_{z z}-I_{x x}\right) r p \\
& N=I_{z z} \dot{r}-I_{z x}(\dot{p}-q r)-\left(I_{x x}-I_{y y}\right) p q
\end{aligned}
$$

where $X, Y$ and $Z$ represents the external force components (longitudinal, lateral and vertical); $L, M$ and $N$ are respectively, the roll, pitch and yaw moments; and $I_{0}$ corresponds to the moments and product of inertia of a rotating body. The kinematic relation for the pitch rate and roll rate about $Y$ and $X$-axis are written as:

$$
\begin{gathered}
\dot{\theta}=q \cos \phi-r \sin \phi \\
\dot{\phi}=p+q \sin \phi \tan \theta+r \cos \phi \tan \theta
\end{gathered}
$$

The helicopter equations of motion are nonlinear, but a meaningful analysis can be employed by converting them into linear differential equations, by considering only small perturbations on a trimmed equilibrium point (represented by subscript 0 ) in the rotorcraft flight envelope. In matrix notation, a 
linearized dynamical model can be seen at the top of the next page (Eq. 9).

Therefore, Equation 9 may also be written as:

$\frac{d}{d t}\left[\frac{X_{I}}{X_{d}}\right]=\left[\begin{array}{ll}A_{1} & C_{1} \\ C_{2} & A_{d}\end{array}\right]\left[\begin{array}{l}X_{1} \\ X_{d}\end{array}\right]+\left[\begin{array}{ll}B_{1} & D_{1} \\ D_{2} & B_{d}\end{array}\right]\left[\begin{array}{l}\Delta \delta_{1}(t-\tau) \\ \Delta \delta_{d}(t-\tau)\end{array}\right]+\dot{x}_{\text {bios }}$

where $X_{l}$ and $X_{d}$ represent the longitudinal and lateral movements. Therefore, the longitudinal movement is expressed by:

$$
\begin{aligned}
& \frac{d X_{l}}{d t}=A_{l} X_{l}+B_{l} \Delta \delta_{l}(t-\tau)++\dot{x}_{\text {bias }} \\
& X_{1}=\left[\begin{array}{llll}
\Delta u & \Delta \mathrm{w} & \Delta q & \Delta \theta
\end{array}\right]^{T} \\
& \Delta \delta_{l}=\left[\begin{array}{ll}
\Delta \delta_{\mathrm{b}} & \Delta \delta_{\mathrm{c}}
\end{array}\right]^{T}
\end{aligned}
$$

Values of interest for system identification are the elements of matrix A (stability derivatives), matrix B (control derivatives), and the delays associated with the aircraft response. Furthermore, the addition of tendency vector, xbias, is constant and unknown. This vector is introduced in the mathematical model to represent measurement errors and noise produced by transducers and instrumentation [17]. Let $\mathrm{J}(\Omega)$ be the cost function, given by:

$$
\begin{aligned}
& J(\Omega)=\sum_{i=1}^{n}\left\|X_{i}^{o b s}-X_{i}^{\bmod }(\Omega)\right\|_{2}^{2} \\
& \Omega=\left(\begin{array}{c}
\frac{X_{u}}{m}, \frac{X_{w}}{m}, \frac{X_{q}}{m}, \frac{Z_{u}}{m}, \frac{Z_{w}}{m}, \frac{Z_{q}}{m}, \frac{M_{u}}{I_{y y}}, \frac{M_{w}}{I_{y y}}, \\
\frac{M_{q}}{I_{y y}}, \frac{X_{\delta_{b}}}{m}, \frac{X_{\delta_{c}}}{m}, \frac{Z_{\delta_{b}}}{m}, \frac{Z_{\delta_{c}}}{m}, \frac{M_{\delta_{b}}}{I_{y y}}, \frac{M_{\delta_{c}}}{I_{y y}}, \\
\Delta \dot{u}_{\text {bias }}, \Delta \dot{q}_{\text {bias }}, \Delta \dot{w}_{\text {bias }}, \Delta \dot{\theta}_{\text {bias }}, \Delta u_{r e f}, \\
\Delta w_{r e f}, \Delta q_{r e f}, \Delta \theta_{r e f}, \tau_{b}, \tau_{c}
\end{array}\right)
\end{aligned}
$$

where $n$ is the number of measurements.

\subsection{Method: Multiple Particle Collision Algorithm}

The cost function to be minimized is a function of the parameters of the dynamic model, such as the helicopter aerodynamic stability and control derivatives, sensor bias, and sensitivities. Therefore, the determination of a parameter vector $\Omega$ that minimizes the cost function given by Equation 14 can be seen as an optimization problem and will be solved by a new meta-heuristics, named the MPCA.

MPCA is a meta-heuristics based on the canonical PCA [18]. This version uses multiple particles in a collaborative way, organizing a population of candidate solutions. The PCA was inspired by the traveling process (with absorption and scattering) of a particle (neutron) in a nuclear reactor. The use of the PCA was effective for several test functions and real applications [19].

The PCA starts with a selection of an initial solution (Old-Config), it is modified by a stochastic perturbation (Perturbation $\{$.$\} ), leading to the$ construction of a new solution (New-Config). The new solutionis compared (function Fitness $\{$.$\} ), and a$ decision is made on whether it can ou cannot be accepted. If the new solution is not accepted, the scheme of scaterring (Scaterring $\{$.$\} ) is used. The$ exploration around closer positions is guaranteed by using the functions Perturbation $\{$.$\} and$ Small-Perturbation \{.\}. If the new solution is better than the previous one, this new solution is absorbed. If a worse solution is found, the particle can be sent to a different location of the search space, such that it enables the algorithm escape from a local minimum.

The implementation of the MPCA algorithm is similar to PCA, but it uses a set with $\mathrm{N}$ particles, where a mechanism to share the particle information is necessary. A blackboard strategy is adopted, where the best-fitness information is shared among all particles in the process. This process was implemented in Message Passing Interface (MPI), looking for application into a distributed memory machine [10]. The pseudo-code for the MPCA is presented by Table 1 .

\section{Results and Discussion}

The computational results obtained with MPCA and GA are presented in Figures 2-5. The GA is codified 
Table 1 MPCA: pseudo-code for the algorithm

\begin{tabular}{|l|}
\hline Generate an initial solution: Old-Config \\
\hline Best-Fitness $=$ Fitness $\{$ Old-Config $\}$ \\
\hline Update Blackboard \\
\hline For $\mathbf{n}=\mathbf{0}$ to $\#$ of particles \\
\hline For $\mathbf{n}=0$ to $\#$ iterations \\
\hline Update Blackboard \\
\hline Perturbation $\{\}$. \\
\hline If Fitness $\{$ New-Config $\}>$ Fitness $\{$ Old-Config $\}$ \\
\hline If Fitness $\{$ New-Config $\}>$ Best-Fitness \\
\hline Best-Fitness $=$ Fitness $\{$ New-Config $\}$ \\
\hline End If \\
\hline Old-Config $=$ New-Config \\
\hline Exploration $\{\}$. \\
\hline Else \\
\hline Scattering $\{\}$. \\
\hline End If \\
\hline End For \\
\hline End For \\
\hline
\end{tabular}

in Matlab Toolbox, and the MPCA was also implemented in Matlab R2011b environment. Computer tests were conducted under Linux operating system, in an Intel Core I5 $2.27 \mathrm{GHz}$. The sinusoidal maneuver is represented by $\delta$ and the results presented take into consideration the average of 4 experiments with seeds generating different random numbers and experimental data generated artificially. The parameters used for MPCA are: 2 particles; 10 iterations (exploration) and the stopping criterion used was the total number of iterations (30).

The red curve corresponds to the real data obtained during the test, the dotted blue one is the result of identification produced by the GA and the results achieved by the MPCA are represented by the dotted curve in magenta. The results shows that especially for $u$ and $w$ there is a slight discrepancy between the measured data and the data obtained by both algorithms. However, for high frequencies there is an improvement in the identification. The results are satisfactory considering the limitations of the uncoupled helicopter dynamic model with six degrees of freedom.

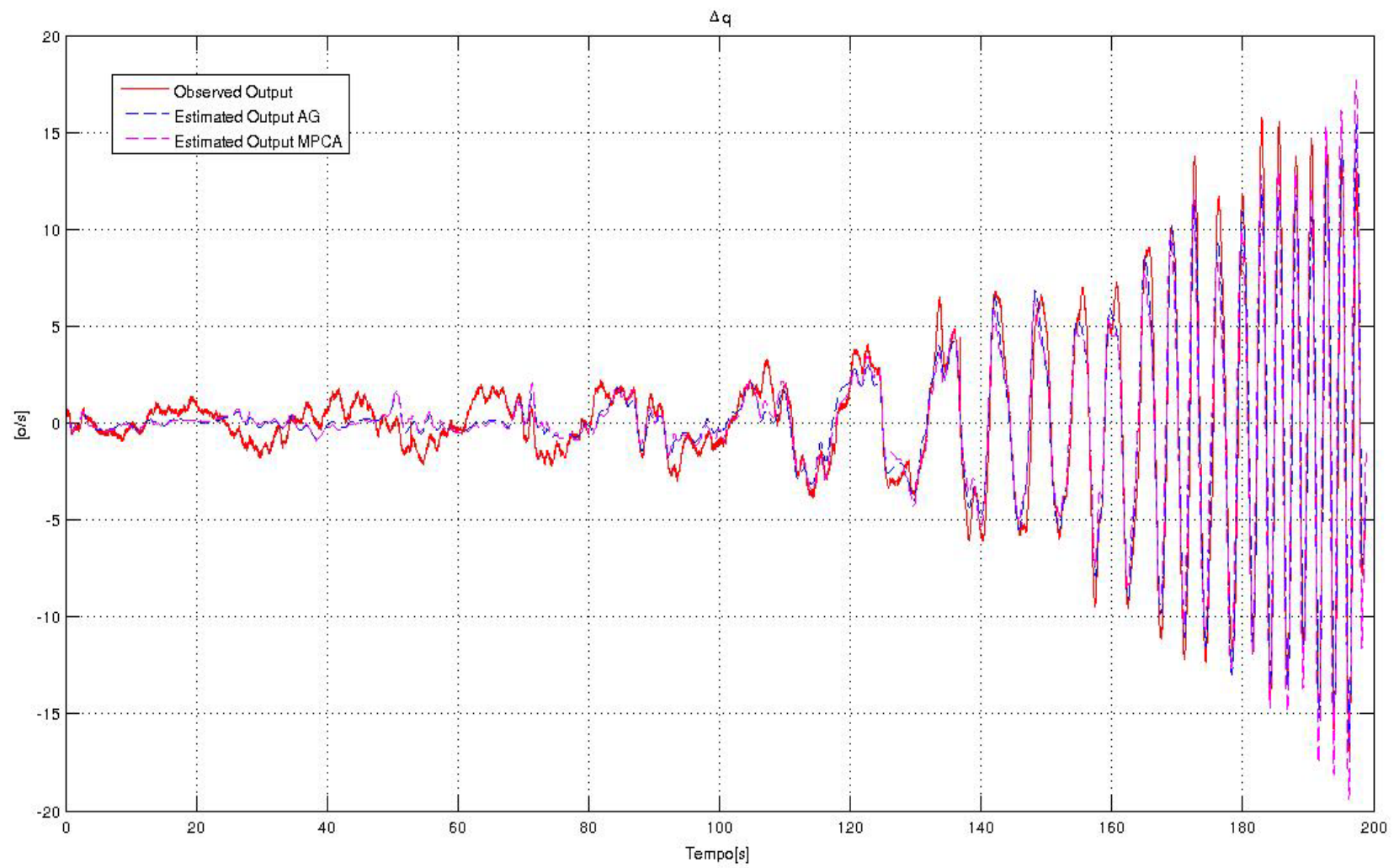

Fig. 2 Pitch Rate. 


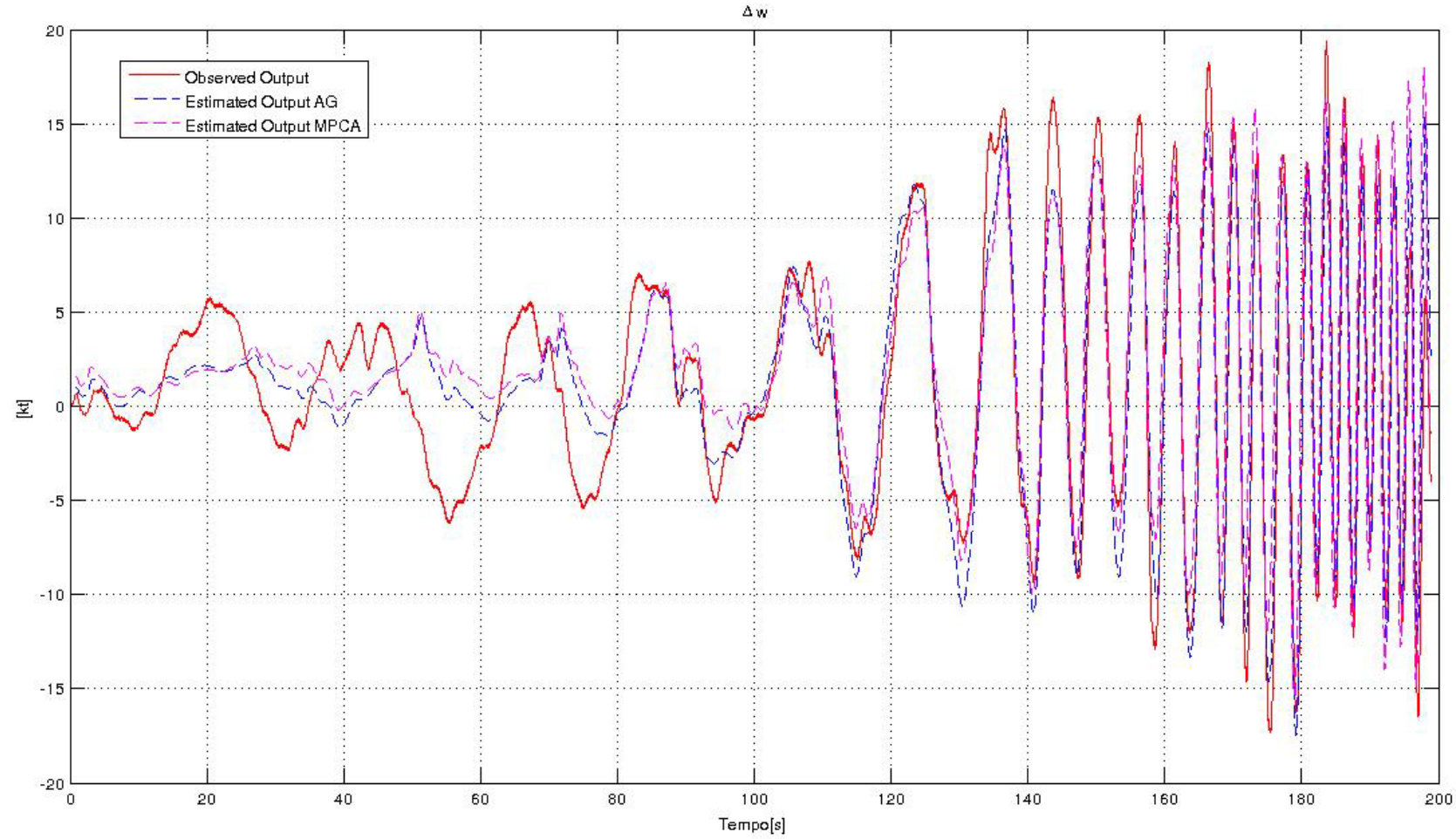

Fig. 3 Vertical Velocity in Body Axis.

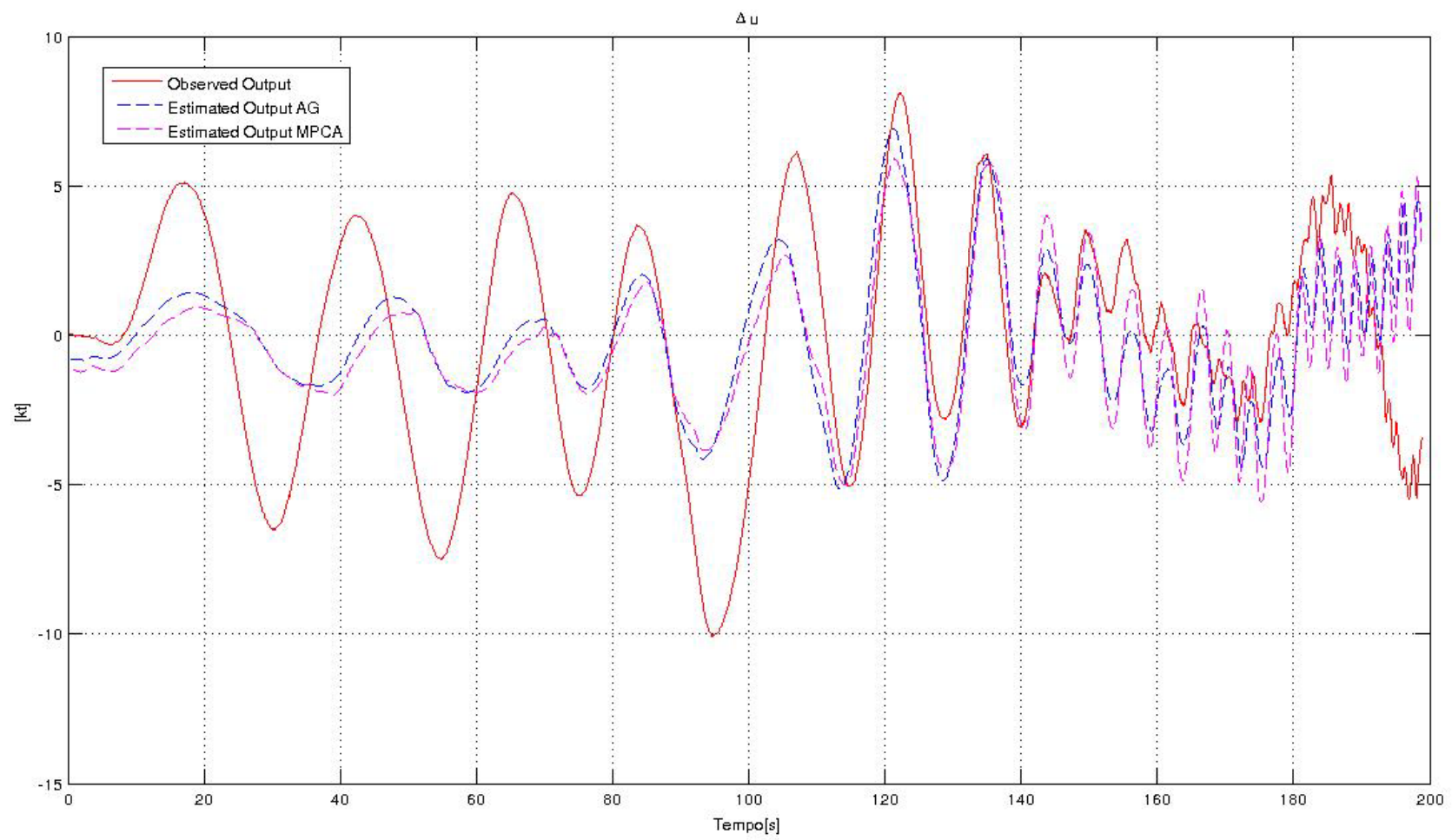

Fig. 4 Longitudinal Velocity in Body Axis. 


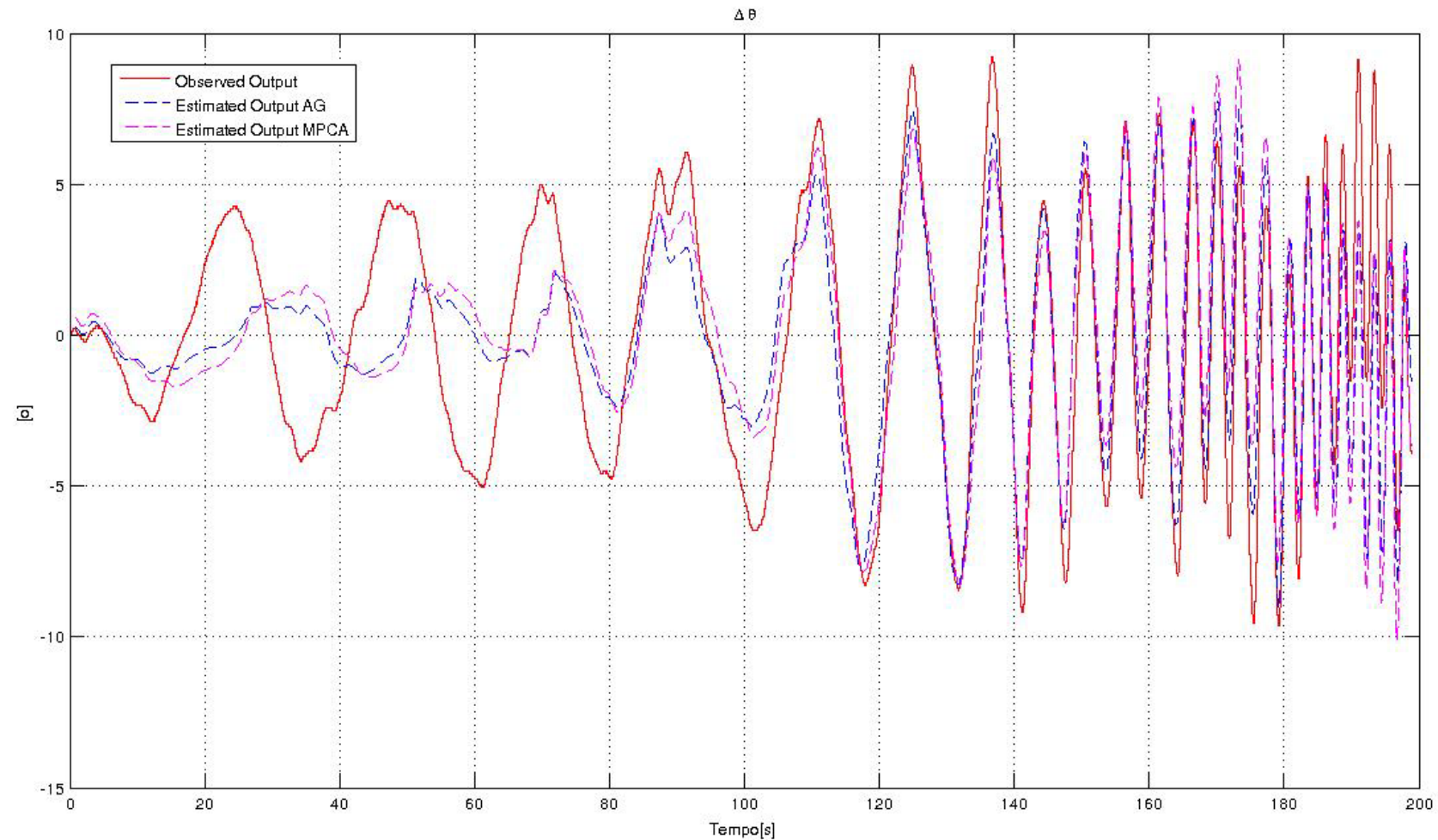

Fig. 5 Pitch Attitude.

\section{Conclusions}

In this work, we compared two stochastic algorithms, GA and MPCA, for a helicopter parameter identification. The techniques were applied only in the estimation of the aerodynamic parameters of the longitudinal motion. The problem is formulated as an optimization process. The algorithms (GA and MPCA) were employed to address the solution of the optimization problem.

The results indicate that GA and MPCA present a good agreement, but it is a little bit better for MPCA implementation. Further work is suggested to apply MPCA in lateral-directional dynamic mode and in a more complex model which includes both longitudinal and lateral-directional dynamic modes.

\section{Acknowledgment}

We wish to thank the partial support given by ITA and IPEV, specially the 2013 class Flight Test Course (EFEV) Students, for providing the experimental data. Also we like to thank CNPQ and CAPES.

\section{References}

[1]. H.F. Campos Velho, F.M. Ramos, Numerical inversion of two-dimensional geoelectric conductivity distributions from electromagnetic ground data, Journal of Geophysics. 15(2) (1997) 133-143.

[2]. E.S. Chalhoub, H.F. Campos Velho, Estimation of the optical properties of sea water from measurements of exit radiance, Journal of Quantitative Spectroscopy Radiative Transfer 72(5) (2002) 551-565.

[3]. S. Stephany, H.F. Campos Velho, F.M. Ramos, C.D. Mobley, Identification of inherent optical properties and bioluminescence source term in a hydrologic optics problem, Journal of Quantitative Spectroscopy Radiative Transfer 67(2) (2000) 113-123.

[4]. G.D. Padfield, Helicopter flight dynamics: The theory and application of flying qualities and simulation modeling, AAIA Education Series, Reston, 1999.

[5]. Agard, Rotorcraft system identification, Agard AR 280, 1991.

[6]. R. Kumar, R. Ganguli, S.N. Omkar, Rotorcraft Parameter Identification from Real Time Flight Data, Journal of Aircraft, 45(1) (2008) 333-341.

[7]. R.V. Cruz, N.S.B Neto, L.C.S. Góes, Preliminary results of longitudinal helicopter system identification using genetic optimization algorithm, 5th Brazilian Conference on Dynamics, Control and theirs Applications, 
Guaratingetá, 2006.

[8]. R.V. Cruz, L.C.S. Góes, Results of longitudinal helicopter system identification using output-error and both genetic and Levenberg-Marquardt optimization algorithm, 19th Brazilian Congress of Mechanical Engineering, Brasília, 2007.

[9]. J. del Cerro, J. Valero, A. Barrientos, Identification of small unmanned helicopter model using genetic algorithms, International Conference on Intelligent Robots and Systems, (2005).

[10]. G.D. Padfield, Applications of system identification in rotorcraft flight dynamics, 13(3) (1989) 207-412.

[11]. E.F.P. Luz, Meta-heurísticas paralelas na solução de problemas inversos, Tese de doutorado, Instituto Nacional de Pesquisas Espaciais, São José dos Campos, 2012.

[12]. R.V. Cruz, Desenvolvimento de um modelo dinâmico para simuladores de helicópteros, Tese de doutorado, Instituto Tecnológico de Aeronáutica, São José dos Campos, 2009.

[13]. R.V. Jategaonkar, Flight vehicle system identification: a time domain methodology, Aerospace Sciences, Reston,
2006.

[14]. R.V. Cruz, L.C.S. Góes, Results of short-period helicopter system identification using output-error and hybrid search-gradient optimization algorithm, Mathematical Problems in Engineering, (2010).

[15]. R.W. Prouty, Helicopter performance, stability and control, Krieger Publishing Company, Malabar, 2005.

[16]. A.K. Cooke, E.W.H. Fitzpatrick, Helicopter test and evaluation, AAIA Education Series, Reston, 2002.

[17]. M.B. Tischler, R.K. Remple, Aircraft and rotorcraft system identification, AAIA Education Series, Reston, 2006.

[18]. W.F. Sacco, C.R.E.A. Oliveira, A new stochastic optimization algorithm based on a particle collision metaheuristic, $6^{\text {th }}$ World Congress of Structural and Multidisciplinary Optimization, (2005).

[19]. W.F. Sacco, D.C. Knupp, E.F.P. Luz, A.J.S. Neto, Algoritmo de colisão de partículas, Técnicas de Inteligência Computacional Inspiradas na Natureza Aplicação em Problemas Inversos em Transferência Radiativa (2009). 